\title{
Take Your Time First, Time Your Search Later: How College Students Perceive Time in Web Searching
}

\section{Authors}

Shu-Yi (Max) Chen

School of Information, University of Michigan

1075 Beal Avenue, Ann Arbor, MI 48109-2112

Email: maxchen@umich.edu

Soo Young Rieh

School of Information, University of Michigan

1085 S. University Avenue, Ann Arbor, MI 48109-1107

Email: rieh@umich.edu

This study explores people's perception of time during their Web searches. Time is a major component of the context for information behavior, but in empirical studies it has been implied rather than investigated explicitly. The data were collected from Web search experiments in which participants were asked to conduct searches on three given tasks under differing search time conditions. The paper reports on findings drawn primarily from the exit interviews of 45 undergraduate and graduate students on their perception of time in Web searching. Study results indicate that at the beginning of their searching activity, participants did not explicitly consider temporal issues. However, these issues usually surface with the passage of time, especially when searches fail to go as planned. Perception of time is closely entangled with familiarity and difficulty of search task. In general, participants enjoyed spending time in searching and were not excessively concerned about time constraints. On the other hand, participants' affective experiences were sometimes caused by temporal issues. In conclusion, the study results indicate that temporal issues interlace with other contextual and affective factors in the process of Web searching.

\section{Introduction}

Time is an essential component in all human activities and is also a major part of the context 
in information behavior (Savolainen, 2006). Traditional information studies, however, have focused only on "seeking without consideration of connections across time" (Solomon, 2002, p.229). As Savolainen (2006) pointed out, temporal factors are more likely to be implied in information behavior studies and less likely to be discussed explicitly because "all human action is embedded in time" (p. 111). Consequently, there is little understanding about how people perceive time when they look for information. Not only does information seeking go through different stages in time (Kuhlthau, 2004) but also the search for it in real life is one that evolves over time (Bates, 1989). Even though time, being a contextual factor, must play a role in and exert an impact on information behaviors, what is known about information behaviors more resembles pictures taken at a specific time in history than real behaviors changing along the timeline (Dervin and Nilan, 1986).

This paper therefore directly explores temporal issues by analyzing interview data collected from an experimental study on time pressure in Web searching, addressing the following questions:

1. How do people generally perceive time in Web searching?

2. To what extent do people estimate and allot time for searching before they begin their searches?

3. What do people experience with regard to temporal issues during their Web searches?

With empirical answers to the above questions, it should be possible to characterize the role that time plays in information seeking behaviors.

\section{Literature Review}

While calling for a turn to the user-centered approach, Dervin and Nilan (1986) suggested that researchers pay more attention to temporal issues given that information behaviors are not static or immobile. For the past two decades, many studies in the information behavior field have at least implicitly identified time as one of the contextual factors, especially studies such as Kuhlthau's (2004) stages that view information seeking as a process. Some views separate time from context and treated time as a background or basic issue. For example, Courtright (2007) examined concepts of context for information needs, seeking, and use, but did not include time as one of the contextual factors. Nevertheless, Courtright suggested longitudinal research in speculating that "a relational and dynamic view ... might emerge" (p.293).

Though many information behavior researchers may have included time in their research, it is clear that only a few have explicitly discussed or defined temporal issues. In defining affective 
load for Web searches, for example, Nahl (2004) included and defined time pressure as one of the components, stating that time pressure would intensify uncertainty and in turn increase affective load, which, if too high, might cause a user to give up on a search task. Time pressure thus plays an evident and important role in Web searching. Despite including time pressure as a factor, however, Nahl was more interested in its impact on Web searching than in the concept of time pressure itself.

Savolainen (2006) reviewed several studies that explicitly discussed temporal factors as context, especially in everyday life information seeking, identifying three approaches including, from most to least abstract, temporal factors that are attributes of situations, qualifiers of accessibility, and indicators of the information seeking process. The first approach is to the general time concepts surrounding life and situations therein. The second approach is treated as a constraint in the form of time pressure imposed on access to information sources. The last approach is to viewing time as a qualifier in the information seeking process. Among other findings, Savolainen suggested that temporal issues can be treated as "time affordance," meaning the time available for seeking and using information (p.123). Temporal constraints could be viewed either subjectively or objectively and will, for example, affect the source and channel selection of information seekers. The literature that Savolainen reviewed focused more on the contextual nature of temporal issues and less on how people perceive time.

On the other hand, McKenzie and Davies (2002) suggested a constructivist view of time, claiming that "When an individual seeks information is as important as what he or she seeks" (p.4), because the timing issue affects how that individual makes sense of his or her situation. This idea they developed was from Dervin's (1992) "time-space-bound" concept for individuals in the belief that it would constitute an approach that allows different time worlds, or representations of time, for individuals. That approach is more in line with the philosophical assumptions behind many qualitative methods adopted in the LIS field and could certainly be applied in the examination of differing human perceptions of time.

Temporal issues are also considered along with tasks and information seeking. Li \& Belkin (2008) proposed time as being one of the generic facets of tasks, with sub-facets that include frequency, length, and stage. Length is the span of time it takes to finish a task, and stage is the completed phase in a task. The above classification provides a promising map for studying temporal issues in tasks, and more work based on these facets is accordingly expected.

In sum, very few studies directly focus on temporal issues in the information behavior field, especially on how people perceive time in the process of seeking information. This paper 
intends to fill the gap by characterizing people's perception of time when they conduct Web searching.

\section{Research Design}

This paper reports one part of the results from a study that investigated students' Web searching behaviors under time pressures. The data were collected from an experimental study by means of search logs, questionnaires, and interviews. Participants were asked to finish three Web searching tasks and then complete three post-task questionnaires, one after each task, and one exit interview at the end of the experiment. The results reported in this paper are primarily based on the exit interviews. The data analysis from search logs and questionnaires will elsewhere be reported.

\section{Data Collection}

The experiments occurred from April to June 2008 at a research university in the United States. The procedure of the experiments was as follows. Once a participant signed a consent form, he or she completed a background questionnaire which inquired about demographic information along with general Web search experiences, satisfaction with search results, and confidence in the ability to find information on the Web. The participant was then given the first search task and was asked to estimate the time needed for searching on the task. The participant was able to use the time he or she requested to complete the first search task. After conducting a search for the first task, the participant was asked if he or she wanted to modify the estimated time for the second search task, which was similar to the first task. When the participant responded to the experimenter with the estimated time needed for the next similar task, only half of the time that was given for the second task. In this design, time pressure was assumed to arise given that it equals time required divided by time available (Hendy et al., 1997). For the third task, the participant was given all of the time that he or she needed for the second task. The three search tasks used in this study were drawn from the complex, interactive QA (ciQA) topics at TREC 2006 (Kelly \& Lin, 2007). Out of five ciQA topics, three were chosen based on consistency of topic style and the perceived difficulty of topics. Participants' searching activities with respect to the three tasks were recorded with TechSmith Morae, a software capable of capturing everything shown on the computer monitor along with logs of all actions 
(keystrokes, mouse-clicks, Web page changes, etc.) performed by the participants. Each time a participant completed a task, he or she completed a post-task questionnaire which addressed task difficulty, perceived success, confidence, and feelings of being overloaded. When all three tasks and the post-task questionnaires were completed, an exit interview was conducted. The purpose of this semistructured interview was to better understand the participant's perception of time with regard to the Web searching experience in general as well as the search tasks he or she had just completed in the experiment. Total duration of the study for participants ranged from 40 to 90 minutes, depending on the estimations of time needed to complete the tasks given.

\section{Sample}

Forty-five undergraduate and graduate students volunteered to participate in the study in response to flyers posted around campus. Among the 45 participants, 32 were undergraduates and 13 were graduates. The 25 females and 20 males came from diverse academic backgrounds including the social and natural sciences, literature, engineering, and business. On average, the participants reported that they spent 9.9 hours a week in Web searching. Thirty five of the 45 participants (78\%) reported searching on the Web twice or more times daily. Moreover, 41 participants (91\%) reported that they were always or mostly always able to find the information they wanted on the Web, while 42 (93\%) were somewhat or very satisfied with the results of their Web searches. Thirty nine of the participants (87\%) were somewhat or very confident in their ability to find information on the Web.

\section{Data Analysis}

This paper reports on the analysis of exit interview transcripts in which temporal factors with respect to people's Web searching experiences were broadly discussed beyond the three tasks given. The content analysis approach was employed to identify major themes of time perception as well as to characterize temporal issues and considerations that participants experienced with their Web searching.

\section{Results}

The results of the participants' perceptions of time are presented in three categories that 
address three research questions respectively. The first category presents common, taken-forgranted postulates and strategies employed by participants in Web searching in general on temporal issues, such as that information was quick to find on the Web. These notions then laid the basis for the second category, which includes the presumptions that participants made before they undertook a search task, such as the time they needed for a certain type of task. The third category is about experiences drawn from the entire searching process, including, for example, decisions participants made, actions they took, and lessons they learned during the search.

\section{Research Question 1: How do people generally perceive time in Web searching?}

\section{Time taken for Web searching}

Data analysis revealed that the participants' perception of time is mixed in terms of time needed or taken for Web searches. While some participants responded that they could find information on the Web in a relatively short time, saying "you find the majority of information right off the bat" (S22) or "usually I think it will only take a minute or two" (S28), ten other participants said that they put a great deal of time into searching. Participants attributed such behavior for at least two reasons. One reason was that they simply searched until they found something of value. Another reason was that they tended to search when they had nothing else to do or when they felt no time pressure. That is, some participants conducted Web searching when they did not need to think too much about time, and naturally this affected how they thought or didn't think about time. Some examples of searching until something was found included:

- I spend quite a lot of time actually, until I get the results. (S38)

- I just kind of go for it and however long it takes, I just kind of stay there because I spend a lot of time on the computer anyways so I usually don't keep track. (S40)

As mentioned, some participants conducted Web searching when they had more time available.

- I usually search for stuff just when I don't have much else to do. (S01)

- It's not like oh my God I have 5 hours to research about this topic and I have 
to do it. I never have to do that. Just more relaxed. (S35)

Time used for Web searching and work tasks

Notably, most participants did not always clearly distinguish the amount of time used for work tasks and search (sub-) tasks. They tended to perceive time from a broad work task perspective rather than from consideration of temporal issues in Web searches of their own. This outcome showed that the specific search result a user seeks is determined by a current task-related goal (Xie, 2000), thus making it difficult for participants to separate out a search task from task-oriented work in terms of time used for each. The following quotes demonstrate how search tasks and users' task-related goals are closely linked with respect to time perception.

- I would say that I give myself an estimated time of completing my task that is related to the search. For example, if I'm writing a paper or I'm doing something at work I'll say that I have to have this complete thing like writing the paper done by this time or I need to perform this chi-square analysis by this time so then like you know it doesn't matter if I use more time searching and less time writing or less time searching and more time writing as long as it's done by that time. (S15)

- I estimate how much time I think the whole, total paper will do and I kind of gage like okay l've been researching for this amount of time I need to get started writing on it. But I don't really set a ... when I find enough information to write is kind of when I'm done searching. (S31)

A related finding is that some participants deferred part of the assessment of information for the future and didn't feel that reading time should be counted as a part of the searching.

- I need time to read and understand the content [but] not to look for that information. I can reach there very fast but I need time to figure out is it useful or not. (S02)

- Maybe for once I can search for a whole day and copy a lot of content, maybe 10 pages, 12 pages, and then the next day I will look at the content I copied and I delete the content I don't want. So I will find the content first then I decide which content I need. (S03) 


\title{
Research Question 2: To what extent do people estimate and allot time for searching before they begin their searches?
}

\author{
Estimation of time needed for Web searching
}

Most participants (about 75\%) tended not to estimate how much time they needed, at least not explicitly, when they began Web searches. This can be explained by two reasons, one of them being that they might simply not have thought about time given their confidence that their Web searching would be completed rather quickly, as shown in the following quotes.

- I usually just go for it. Like I said if it's something common enough or something that exists it usually pops up quickly enough that I don't ever have to worry about time. (S22)

- Depending on what the topic is I sort of mentally designate like oh this will take two seconds or whatever but I don't really think about that [time]. (S20)

Another reason for not estimating is because it is simply too difficult to estimate the amount of time needed to find information. S14 and S15 below explain why they did not or could not estimate the time.

- It's really hard for me to do that because it's hard to know where the information is going to be or if I'll be able to find it so l'll try to estimate it but not always and usually that's not really that effective. (S14)

- I don't really think about how much time l'm going to be spending on the Internet searching for it before hand. And I think the reason why is you never know how much there is. (S15)

However, there were still six participants who said that they did estimate the time needed accurately based on their prior experience, as illustrated by S24.

- I never time myself to see how long l'll get it but depending on how confident I am about the information I often like just know how long it will take me just because I've been doing it for so long. (S24)

Participants learn from their experience to estimate the time needed for a variety 
of search tasks. The data analysis revealed that estimation of time needed is often associated with the familiarity of the search tasks involved. Less familiar tasks perceived by participants were estimated to require more time. The following two examples indicate how familiarity with the task and estimation of time closely relate.

- Sometimes if it's something I feel like I know a lot about or if there's a lot of information about then I would estimate less time like oh this won't take me that long. But if it's something that I don't know anything about and it seems really complex then it will probably take a lot longer. So I guess in a vague way I do [estimate] but I don't usually think like it will take 2 hours to do this. (S34)

- It depends on the situation. [...] If I can expect the answer or I'm much familiar with the question I can find it much faster when I do the search but if the question or the answer is not what I expect or it's not what I'm familiar with I feel how can I take so long. (S04)

Similarly, estimation is associated with the different types of search tasks involved. Participants differently estimated the time needed for school-related searches, such as for term papers or other homework. Some said it usually took longer, some say shorter. For self-interest searches, however, participants thought that it usually took less time to locate the information.

- So if I want very specific information for my homework or some research I need much more time than $10 \mathrm{~min}, 5 \mathrm{~min}$ of course. (S02)

- But everyday stuff I would probably underestimate. (S26)

- I know if I'm going into a big research paper that I should plan maybe an hour to do research whereas if I'm just looking up something smaller like hockey scores from 1994 I could probably find that a little bit more quickly. (S18)

\section{Allocation of Time for Web searches}

As discussed earlier, most participants in the study tended not to think about time when they started searching. Consequently, no explicit limit or allowance of time was set during the searches. Only a handful of participants, such as S11, planned for duration before they started searching. The following examples demonstrate 
how some participants mentioned the time allotted for Web searches.

- I usually just keep searching until I'm done. If it's for like a report or if it's something I'm researching for for over half an hour I never really give myself a time limit. I will just keep searching until I'm satisfied. (S19)

- [...] that's why usually when I do my own searches I don't put any time constraint in it. I just search for it. I find that it's a bit hard to work under time pressure yeah. (S38)

- [If I want to search for something I may say oh it may take me an] hour or two hours because I have other work I need to check together so if I search papers I will tell myself maybe today I will only search for two hours and that's the two hours I may write something or I may do other work but if tomorrow I have other time I can check the...research the topic again. (S11)

Allotment of time is associated with participants' estimation of time needed for a task. Therefore when participants attempted to allot time for searching, they were also affected by their familiarity with the different types of search tasks involved. Participants allotted different amounts of time for different types of search tasks and usually allotted more time for school-related searches.

- Usually when I'm doing searches it's for a paper or something and I'm usually pretty good about giving myself enough time to write those kinds of things or do a lot of research on them first. (S31)

- I think sometimes it depends on the topic you search. I think sometimes if it's a topic you never searched before it maybe have a lot of resources...and then you say I need to spend more time. But if the topic I've already searched for two or three times or more, several times, I just check the topic again and there are not so many new paper so I just stop, just half hour. (S11)

- But for my own interest I think I can use unlimited time. (S05)

\section{Research Question 3: What do people experience with regard to temporal issues during their Web searches?}

Implicit comparative perception of time

Virtually all the participants in this study estimated or thought about time implicitly rather than explicitly. When they tacitly compared estimates of the actual amount 
of time used, they internally perceived it to be longer or shorter. This is where the implicit estimation makes its entrance. Some examples include participants' use of phrases such as "Ionger than I thought" or "faster than I would expect," though they did not actually "think" or "expect" when they start searching. Hence time was an issue only implicitly considered and was rarely noticed by participants until they experienced time lapses.

- I can't think of anything specific but l'm sure there have been plenty of times when it's like wow that was really quick or this is taking a lot longer than I thought. (S31)

- I guess more often than not I find information faster than I would expect. (S08)

- Maybe subconsciously [I estimate how much time I think something might take]. I don't think I usually sit down and say alright this search will take me five minutes. (S18)

\section{Losing track of time during Web search}

Several participants mentioned that they tended to lose track of time when searching for information on the Web. They felt that the time flew during their searches, and they often ended up spending more time than they expected. Two of the reasons for greater than expected amounts of time used include that they were following the links or that they are on the verge of discovering something interesting.

- Like I've definitely done searches where l've found something interesting so I go looking into more and click more links and then the next thing I know an hour or two has past and I'm like I totally don't remember spending that much time for it. (S40)

- My searching is initiated by my own interests so you know doing something you like ... when we're doing something we like we don't know how much it takes [...]. Yeah enjoy. I enjoy searching my own interests. (S10)

Another reason for participants spending more time on recreational searches was that they did other things between Web searches, a fact that might have made it difficult to talk clearly about temporal issues in their searching alone. 
- But basically I'Il just switch between topics for recreational purposes whereas the other one I'm usually focused on the task at hand. So that's just because it's recreation versus work. (S21)

- I would say usually when I have a lot of time, there's a lot of Facebook and checking my email added into those like in between searching, a lot of procrastination while I'm actually searching. (S31)

Affective experience from Web searches

Among the differing experiences, searches that take longer tend to induce negative affective responses such as frustration, in participants. For instance, S41 mentioned that she used frustration as an indicator of having spent too much time because she did not keep track of the clock when she was Web searching.

- I think I don't intentionally estimate but when two hours past I just don't have patience on that searching anymore. (S07)

- (when asked how she knows when it is taking too long) I think it's never really an actual measure of time. It's just an amount of frustration that ... (S41)

However, if participants failed to find the desired information during the estimated time, they then decided that the information might not in fact be there, leading them to set a time limit on their searches.

- Generally l'm pretty open-ended at least when I'm starting and then after I've been at it and been frustrated for a little while I start setting time limits on if I can't find it. (S39)

- But as I told you onto 15 minutes if I cannot find the information I will give up. (S02)

\section{Discussions}

From the results, it is clear that time was a contextual factor in Web searching. Additionally, participants perceived time differently in different search stages. Participants in this study thought less about time when they started searching, but began to think more about it as time passed. They also combined time taken across different events, such as in search and work 
tasks. Moreover, their perception of temporal issues as related to Web searching affected their behaviors and decisions in searching, such as in their failure to consider time limits. These and other points fairly answer the research questions of this study. Some discussion follows.

\section{Temporal issues emerge mostly when Web searching is not going well}

People seldom explicitly estimate time before beginning searches. Participants in this study either thought the time would be short, or they searched when they had plenty of time. So when they obtained the information they wanted quickly enough, temporal issues did not command their attention. However, when they spent more time than they tacitly expected, either for failure to find their information quickly enough or simply due to overindulgence with Web surfing, temporal issues tended to arise. Participants thought it reasonable not to plan or allot time in advance when they thought Web searches would not take long to conduct. This, however, only increased the difficulties they encountered in exploring temporal issues during Web searching. When time is allotted for searching, to be sure, it can well reveal how people estimate time when Web searching.

The foregoing suggests several directions for future research. First, the research method requires careful design to allow temporal issues to emerge from the background. Second, a retrospective method of collecting data may be necessary. Third, as time, especially at the starts, is not explicitly considered, some implicit measurements may become necessary to determine how people perceive time when searching the Web given that they may be unable to talk about it sufficiently. Time allotted for searching, for instance, could be used to gauge how people estimate the time needed for their search tasks.

\section{Perception of time in Web searching is subjective}

When participants sense the existence of temporal issues because they feel their searches take too long, those issues may remain subjective in nature. To decide whether or not a search has taken too long, a person has to estimate, possibly implicitly, the time needed and then compare it to the time actually used. However, people may estimate differently for the same task due to dissimilar experiences in the past or familiarity with the task. Actual time used by different people may not be alike, either. Further, because people sometimes do not keep track of time used, there is in fact no "actual time" but rather only time sensed in the search. 
This sensed time length is then used for comparison with estimated time. Clearly, in this case, both times are subjective.

For example, one person could unconsciously estimate that a task will take 5 minutes to complete, while another person will estimate it to be 15 minutes. Thus if in both cases the search actually takes 20 minutes, the two will feel differently about the results. Moreover, should the search take 10 minutes, temporal issues may never come to mind in the case of the latter person. What if the $\mathbf{1 0}$ minutes feels like $\mathbf{3 0}$ minutes for this latter person? Therefore, temporal issues can be subjective even when they are explicitly presented, leading to the conclusion that the subjective method of measuring time should be considered in studying Web searching. These issues will inevitably complicate investigations to come.

\section{Temporal issues in Web searching are entangled with other issues}

As indicated by the results, participants in this study sometimes could not separate searching itself from larger work tasks or other constraints when considering temporal issues in Web searching. Moreover, some participants considered reading time to be a part of searching time, while others believed that searching is accomplished after they have skimmed through search result pages and located relevant websites (it should be noted that reading time does not count toward searching time). Equally important, some people conduct their searches concurrently with other activities (e.g., email checking), while still others fail to keep track (or lose track) of time.

These findings echo Solomon's (2002) when he suggested that information seeking, or "discovery of information," should be regarded as "being constructed through involvement in life's activities, problems, tasks, and social and technological structures" (p. 229). Thus not temporal issues alone should be taken into consideration in information studies; they should be explored together with other contextual issues to obtain a more holistic picture.

The findings of the study, however, greatly complicate the research on temporal issues in Web searching. Further, this difficulty could well arise in a number of other time-related studies of information behaviors. Because people can commonly perform or think about several activities simultaneously, it could prove difficult to discover any clear boundaries between two events and thus complicate the 
investigation of temporal issues in information behaviors. One approach that is possible to take is to employ more creative qualitative data collection and analysis strategies to probe more deeply into the actual meanings behind descriptions about time.

\section{Time needed is closely related to the perception of task}

Participants in this study thought of time with respect to task difficulty or familiarity. For example, familiar or simple tasks required less time, while unfamiliar or complex tasks required more time. That is, a familiar or simple task went more quickly than an unfamiliar or complex one, to their minds. Participants also responded by allotting different amounts of time for different types of tasks. Thus, one method by which they perceived tasks was the time needed for a task. It will be interesting indeed to delve more deeply into how people think of tasks in terms of temporality, as this would certainly affect how they choose their search strategies, which in turn affects the design of information retrieval systems. For example, people may want to allocate "enough" time for work-related search but may be unwilling or willing to spend more time on searches related to their own interests.

\section{People may lose track of time in Web searching}

A few participants mentioned that they spent a lot of time searching, enjoyed it, and did not comprehend how the time went by so quickly. This suggests the "flow" experience proposed by Csikszentmihalyi (1988a), in which he stated that the optimal experience "obtains when all the contents of consciousness are in harmony with each other, and with the goals that define the person's self" (p.24). In the Web environment, Chen et al. (1999) studying flow experience in Web users found that it exists for a number of Web activities. Due to connectivity in the Web environment, it is possible that a Web search could turn into Web surfing unrelated to the original search, especially when people find themselves to be in this kind of optimal experience.

More pointedly to temporal issues, Csikszentmihalyi (1988b) stated that "a 'distorted' sense of time" is often perceived in flow experiences, leading people to feel that "hours seem to pass by in minutes" (p.33). One participant talked about this in a similar fashion: "I know an hour or two has past and I'm like I totally don't 
remember spending that much time for it" (S40). Pace (2004) has suggested that attention paid to surfing but not to time could explain such distortion. Clearly people have flow experiences while searching on the Web, in which "[t]he clock no longer serves as a good analog of the temporal quality of experience" (Csikszentmihalyi 1998b, p.33). This not only supports the subjective view of time reported previously but also creates challenges for the study of temporal issues in Web searching, especially of those individuals who experience "flow". Content analysis and grounded theory approaches were accordingly suggested for studying flow experience in Web users (Chen et al. 1999; Pace 2004), and additional research on how temporal issues under flow conditions affect Web searching can be expected.

\section{Affective experience can result from temporal issues in Web searching}

Some participants in this study expressed feelings of frustration when they failed to find information quickly enough or in advance of the time limits they implicitly estimated. This result aligns with Nahl's (2004) affective load study in which she measured time pressures by "subtracting 'Felt Length' from 'Expected Length'” and then posited that when time pressure is high "a user is affected by frustration [and] even rage" (p.193). However, participants in this study did not communicate the idea of pressure caused by time, instead indicating that they felt frustrated when they could not readily find the information they wanted. This could well prove to be a terminology issue, as many studies of time pressure define it to be time required divided by time available, a concept of ratio rather than of difference (e.g. Hendy et al. 1997). Others define time pressure as the amount of information processed in a fixed period of time (e.g. Payne et al. 1988). The difference method of measurement might be less reflective of the pressure experienced by people under time constraints. However, Nahl's concept of time pressure accurately shows the effects of using a longer period of time than expected on the assessment of people's information behaviors, as seen in this study.

\section{Conclusion}

Though temporal issues have not been systematically studied in the field of information science, they deserve more attention given that they are interwoven with core concepts such as of task and context. By investigating how undergraduate and graduate students perceive time 
when they are conducting Web searches, this study deepens the pool of knowledge on the topic. It shows that temporal issues may in many cases not be planned or even sensed when people initiate a search. Time-related issues are usually accompanied by certain problems, such as the longer amount of time spent in searching and the frustration thereof. Moreover, temporal issues interlace with other contextual factors in Web searches. Finally, the subjective side of temporal issues is frequently to be found in Web searching, such as in the case of flow experience.

Because this paper analyzes only a part of data set from a larger project, the scope is somewhat limited. Additionally, given that undergraduate and graduate students are generally more Web- and search-savvy, the findings of this paper may not be generalizable to shed further light on other groups of people outside of the study. Nonetheless, the implications that this exploratory study advances could well prove helpful for future research. Moreover, future studies can focus on more detailed levels of the search process, such as on one specific search task, and then investigate the work task involved for time perceptions in both search and work tasks, along with actual time used for those tasks. Such research could further support or expand the findings of this study. On the other hand, future studies can focus on broader levels, such as on the general notions about time that people hold not only toward Web searching but also toward information seeking in general, most especially how the perception of time changes along the timeline of an information seeking episode. With the accumulation of more findings on temporal issues, it will eventually be possible to look at information behaviors from more comprehensive approaches. More conceptual and empirical progress can reasonably be expected in the near future.

\section{Acknowledgements}

We thank Raya Samet and Chrysta Meadowbrooke for their research assistance and helpful discussions in conducting the experiments. We also would like to thank $\mathbf{4 5}$ volunteers who participated in the experiments.

\section{References}

Bates, M. J. (1989). The design of browsing and berrypicking techniques for the online search interface. Online Review, 13(5), 409-424.

Chen, H., Wigand R. T., \& Nilan, M. S. (1999). Optimal experience of Web activities. Computers in Human Behavior, 15, 585-608. 
Courtright, C. (2007). Context in information behavior research. Annual Review of Information Science and Technology, 41, 273-306.

Csikszentmihalyi, M. (1988a). Introduction. In M. Csikszentmihalyi \& I. S. Csikszentmihalyi (Ed.), Optimal experience: psychological studies of flow in consciousness, pp. 3-14. Cambridge, UK: Cambridge University Press.

Csikszentmihalyi, M. (1988b). The flow experience and its significance for human psychology. In M. Csikszentmihalyi \& I. S. Csikszentmihalyi (Ed.), Optimal experience: psychological studies of flow in consciousness, pp. 15-35. Cambridge, UK: Cambridge University Press.

Dervin, B. (1992). From the mind's eye of the user: The sense-making qualitative-quantitative methodology. In J. D. Glazier \& R. R. Powerll (Ed.), Qualitative research in information management, pp. 61-84. Englewood, Colorado: Libraries Unlimited.

Dervin, B., \& Nilan, M. (1986). Information needs and uses. Annual Review of Information Science and Technology, 21, 3-33.

Hendy, K. C., Liao, J., \& Milgram, P. (1997). Combining time and intensity effects in assessing operator information-processing load. Human Factor, 39(1), 30-47.

Kelly, D. \& Lin, J. (2007). Overview of the TREC 2006 ciQA Task. ACM SIGIR Forum, 41(1), 107116.

Kuhlthau, C. (2004). Seeking meaning: A process approach to library and information services. $2^{\text {nd }}$ ed. Westport, $C T$ : Libraries Unlimited.

Li, Y., \& Belkin, N. J. (2008). A faceted approach to conceptualizing tasks in information seeking. Information Processing and Management, 44(6), 1822-1837.

McKenzie, P. J., \& Davies, E. (2002). Time is of the essence: Social theory of time and its implications for LIS research. Advancing Knowledge: Expanding Horizons for Information Science, $30^{\text {th }}$ annual conference of the Canadian Association for Information Science, Toronto, Ontario, May 30-June 1, 2002.

Nahl, D. (2004). Measuring the Affective Information Environment of Web Searchers. Proceedings of the 67th Annual Meeting of the American Society for Information Science \& Technology, November 15-19, Providence, RI., Medford, NJ: Information Today. 
Pace, S. (2004). A grounded theory of the flow experience of Web users. Computers in Human Behavior, 60, 327-363.

Payne, J. W., Bettman, J. R., \& Johnson, E. J. (1988). Adaptive strategy selection in decision making. Journal of Experimental Psychology, 14(3), 534-552.

Savolainen, R. (2006). Time as a context of information seeking. Library \& Information Science Research, 28, 110-127.

Solomon, P. (2002). Discovering Information in context. Annual Review of Information Science and Technology, 36, 229-264.

Xie, H. (2000). Shifts of interactive intentions and information-seeking strategies in interactive information retrieval. Journal of the American Society for Information Science, 51, 841-857. 